\title{
The Culture Interpretation of Transformation about Nv Wa
}

\author{
Rui Yang \\ Xi'an College of High Tech, Xi'an University of Science and Technology, Xi'an, Shaanxi, China
}

\begin{abstract}
Mythology is the unconscious artistic processing outcome in human brains. Its producing, developing and changing are close related to the social life of that time. Nu Wa and Fu Hsi appear in mythology, which is the inevitable result that society development reflects in human inner world. When we put $\mathrm{Nu}$ Wa and $\mathrm{Fu}$ Hsi into the society and the time that they were produced, we can discover the human nature that hidden behind the divinity. By researching archeology and literature data, the intricate links between Fu Hsi and Nv wa is revealed.
\end{abstract}

Keywords-Mythology, Nu Wa, Fu Hsi, Primitive society, Subsist

\section{女娲变伏羲的文化学阐释}

杨睿

西安科技大学高新学院基础部, 西安, 陕西, 中国

摘 要 神话是人类头脑中不自觉的艺术加工的产物, 其产生、发展以及变化都与当时的社会生活有着密切的关系。神话中女 娲、伏羲的出现, 是社会发展反映在人类精神世界中的必然结果。文章试图将女娲与伏羲放入其产生的社会时代中, 通过考古资料 及文献资料研究这些形象神性的背后所掩藏的人性的光芒, 这种人性恰恰显现着女娲与伏羲之间千丝万缕的联系。

关键词 神话, 女娲, 伏羲, 原始社会, 生存

\section{1. 引言}

谈到中国神话, 女娲与伏羲是两位极为重要的人物。 在中国神话世界中, 女娲俨如人类善良仁慈的母亲, 她不 仅孕育了华夏民族的生命, 小心翼翼地呵护自己的儿女, 还为自己的儿女开辟广阔的生活空间, 创造了安全稳定的 生活环境。如果将女娲比作慈母, 那么伏羲就可比作华夏 民族的严父, 他教导子民识文字习礼仪, 使华夏走上文明 之旅。女娲、伏羲神秘而又充满魅力, 他们吸引了学者探 索的目光。当我们试着将这两位圣不可偯的大神, 请入那 个遥远又充满野性活力的年代, 他们就变得亲近了许多。

\section{2. 神话中女娲的产生}

马克思说: “任何神话都是用想象和借助想象以征服自 然力, 把自然力加以形象化; 因而, 随着这些自然力之实 际上被支配, 神话也就消失了。”[1] 神话是人类头脑中不 自觉的艺术加工产物, 那它必然有加工对象, 即任何神话 的产生都是有一定的社会文化和事实依据的。

西安科技大学高新学院科研基金项目支持(2013-8)

\section{1 女娲的产生与人类对生的渴望密切相关}

上古时期, 生产力水平极其低下, 这无形中也限制了 人们的自我认识以及对世界的认识水平。此时的人类就如 同一个初涉人世的幼儿, 周遭的一切对他们来说是陌生的、 未知的, 也是充满诱惑的; 他们赖以生存的原始自然环境, 对于他们来说是异常强大的, 任何一种猛兽、任何一种自 然天象都足以使渺小的人类从此消失, 生命在这种环境中 显得那样的脆弱。但是, 恶劣的生存环境并没有吓倒自然 界万年才孕生的智灵, 他们既敬畏自然, 又充满了与自然 斗争的坚定勇气。生命的脆弱促生生存的意志, “生存下去” 成为人们坚定的信念, 于是人类为了生存不断努力、奋争, 一部远古发展史就是一部人类求生的奋斗史。

人们在与自然斗争的过程中, 需要精神上的自我激励 与支持, 所以人们创造出许许多多神话人物, 他们在神话 世界中帮助人们完成与自然界周旋中的夙愿。原始先民独 特的思维方式, 使他们笃信神话人物的存在。那些英雄神、 
反抗神就成了人们反抗自然、追求生存的精神楷模。

正如吴天明所说“求生是全部神话的共通话题”。[2]人 们对生存孜孜不倦地追求直接引发了人们对生殖的崇拜。 摩尔根在研究欧美原始部落时, 从现存的原始部族中发现, 每个部落都有自己的图腾, 这些图滕大都是具有较强生殖 能力的动植物, 人们将其当作自己的血亲, 认为自己是它 们的后代。这正是人们对自身渴望的一种外映, 人们认为 通过虔诚的祭祀, 这种强生殖能力也会降至已身, 从而使 自己的部族长久的生存下去。无独有偶, 在我国出土的大 量史前文物也为我们提供了更确丵有力的证据: 在典型的 母系氏族部落半坡遗址, 出土了大量绘有鱼纹或变形鱼纹 的陶器。鱼, 就是一种具有很强的生殖能力的动物, 所以 受到了此氏族的崇拜。1958 年, 在甘肃省谷县西坪乡, 出 土了一件仰韶文化庙底沟类型的彩陶瓶, 瓶上以墨彩绘有 鲵鱼形动物 (一说人首蛇身或蜓身等)。后来, 考古学家发 现许多这样的鱼纹或变体花纹, 有的学者甚至怀疑此可能 就是女娲形象的前身。

\section{2 女娲形象的文化学阐释}

人类最初的婚配制度（群婚制）决定了人类社会的发 展必将由母系社会开始。在群婚的制度下, 很难发现男性 在衍生后代中的重要作用, 但是女性十月怀胎, 通过分婏 将新的生命带入人世则是显而易见的, 于是原始先民认为 女性在生养后代, 维系氏族种群繁衍生息中起着非常重要 的作用。从考古文物中, 我们也可以很清楚地看到先民这 一观念: 母系社会出土的大量陶像都是女性, 尤以孕妇的 形象居多。在先民眼中孕妇是最美的, 因为在她们浑圆的 躯体中正孕育着生的希望。所以在上古社会早期, 才会在 世界范围内出现女性世系 (母系) 以及女性 (母亲) 崇拜。

正是这种崇拜使女娲以母亲神的形象出现: “俗说天地 开辟, 未有人民, 女娲抟黄土作人, 剧务力不暇供, 仍引 绳于泥中, 举以为人” 《太平御览》卷七八引 《风俗通》), 女娲创造了万物之灵, 她是华夏民族的始祖。从此则神话 中可看出, 原始先民在解释人类的产生时, 将自身的经历 和体验融入其中, 人是由母亲孕生, 所以部族的始祖也必 定是位女性。可爱的先民又用孩童的移情推理, 将女性生 子的理论推而广之, 认为万物也必有其所生, “娲, 古之神 圣之女, 化万物者也。”(汉. 许慎《说文》) 《山海经》中也 说女娲肠化众神, 足见女娲不仅是人之母也是众神之母。

女性世系时期, 女性的社会经济地位也决定了女娲的 母亲神地位。“根据当时人类简单的劳动分工, 妇女是主管 采集经济的, 原始农业就是那时由妇女从采集实践中逐渐 发明的”, “这时人们主要从事采集经济, 妇女负责采集植
物果实及可食的根、茎, 以及监守氏族财产, 教养子女等, 她们的劳动在公社生活里起主要作用”, “由于农业逐渐成 为人类维持生活的必要经济部门, 原始农业的产生和发展, 使妇女在经济上起着重要的作用和占有崇高的地位”[3]。 女性在当时社会中处于领导地位, 体现在神话世界中, 就 是女娲母亲的出现。作为母亲, 儿女的幸福是她最大的心 愿, 所以为了使人类安居, 她又担当起抗击洪水、为民除 害的重任, “往古之时, 四极废, 九州裂, 天不兼覆, 地不 周载; 火爁炎而不灭, 水浩洋而不息; 猛兽食颛民, 鸷鸟 算老弱。于是女娲炼五色石以补苍天, 断鳌足以立四极, 杀黑龙以济冀州, 积芦灰以止淫水; 苍天补, 四极正, 淫 水涸, 冀州平, 狡虫死, 颛民生”(《淮南子. 览冥训》)今日 当我们读这则神话时, 母亲的伟大以及先民对母亲的崇敬 和爱戴, 依然清晰可得。母系氏族社会内, 事无巨细, 均 由族长一一位年高德重的老母亲来定夺, 氏族中的婚配 理当是由“母亲”来操心, 时至今日在儿女婚事上依然是母 亲在操持着。那么母亲神女娲更不可能免去此职, 古书记 载她还创制了笙簧, 建立了婚姻制度, 成为了中国古代的 第一位媒神。

原始思维决定了神话对于原始先民来说, 是自己生活 真实的再现, 因此, 可以说神话是在曲折地反映着当时人 与自然、人与社会、人与人之间的种种关系。这种女尊的 社会氛围（母系社会）必然会反映在神话中, 即此时出现 的神话人物都是女性神。据学者研究在国内外都较普遍存 在过史前女神崇拜现象, 在以女性为中心的社会中, 女神 的出现是必然的。吴天明在其著作《中国神话研究》一书 中, 使用详实的材料和严谨缜密的推理, 论证了中国诸神 前身是女性神的可能性。此外, 当时社会的分工尚未明细 化, 所以我们看到女娲身负的神职非常之多：创世神、始 祖神、媒神, 这也是与当时女性在社会中担任的角色和分 工甚多相关吧。

\section{3. 神话中伏羲的产生以及文化学阐释}

自从人类拿起工具的那一刻开始, 就注定了社会必将 以它特有的规律向前发展。随着畜牧经济和农业种植的发 展, 深耕农业代替浅耕农业, “男子在氏族中才占主要地位。 因为深耕必用笨重的农具掘松土地, 这不是妇女之力所能 胜任, 于是男子渐渐扩大其经营范围。更进一步, 男子掌 握了农业生产, 妇女变为辅助者。于是男子长期居家经理 财富, 男子地位跃居首位; 妇女反而退到第二位”[4]。生 产力的发展, 人们的分工产生了重大的变化, 男性在农业 和手工业这两个社会主要生产部门中, 逐渐成为主要劳动 力; 女性则从事纺织及家务等次要的劳动。这种转变是历 史性的, 它必然会给社会各方面带来震动: 社会经济结构 
的改变使男女在经济生活中的角色发生了戏剧性的对换, 从而彻底颠覆了女性主导的社会生活结构。社会经济的主 要生产者既然已不再是女性, 那她们也就随之逐渐失去了 高高在上的社会地位。同时, 在种植和饲养家畜的过程中, 人们对生殖有了新的认识, 人们发现了“种子”在生物繁殖 过程中的巨大意义, 这无形中也帮助人们重新认识自己。 母系氏族鼎盛时期人类开始实行对偶婚, 这种婚姻制度的 出现, 改变了母系社会伙婚制下 “只知其母不知其父”的现 象, 人们渐渐开始明确父子之间的关系, 人们发现男性在 生殖中的作用, “在把包括人在内的万物丰产视为最高需要 的文化里, 男子的生殖作用一旦确认, 这对男子的地位来 说, 该是多么重大的转折”[5]。社会生产力发展促进经济 增长的同时, 也促使私有财产的出现: 房屋、家畜、土地 逐渐私有化。人类的私欲是种本能, 他们总是尽可能多的 将自己的财产遗留给自己的后代, 而不愿被同族其他人分 割。所以在父亲身份日益确定的情况下, 在子女对财产继 承需要的推动下, 女性世系向男性世系转变[6], 父系社会 开始登上历史舞台。

世变时移, 男性逐渐开始在社会生活各个方面占据主 导地位, 这就促使精神领域的统治者也必然有所改变。正 如前文所述, 整个原始社会时期, 人类的共同目的就是求 生存, 男性在生衍后代中重大作用被发现必然会导致男性 崇拜的出现, 所以在中国乃至世界古史中, 继女性及女性 生殖崇拜之后, 都出现过男性及男性生殖崇拜。

男性崇拜要求在精神世界中重塑男性的地位, 同样要 求在神话世界中重塑众神的地位, 甚至众神的性别。所以, 开天辟地的大神盘古变成了男性, 婚姻之神变成了白胡子 月老, 门神由原本代表女性的郁垒、神茶两种花, 变成了 威风凛凛的男性......诸女神纷纷向男性神转变。但是, 母 亲的功绩是永远无法磨灭的, 是母亲孕育了人类, 人类对 母亲的情感会永远激荡在历史长河中, 日久弥深。即使父 系社会神话世界中的大神——伏羲也注定是由母亲所生: 其母华胥氏在“雷泽”踩到了巨人的脚印, 有所感动, 而孕 生伏羲 (《太平御览》卷七八引《诗含神雾》说: “大迹出 雷泽, 华胥履之, 生密犧。”) 。

女娲给与了华夏族人 “形”, 伏羲则给与了华夏族人 “灵”, 他是华夏文明的缔造者。在迈向文明的征程中, 是 伏羲引领华夏先民告别洪荒, 走向文明。“古者包犠氏之王 天下也, 仰则观象于天, 俯则观法于地, 观鸟兽之文, 与 地之宜, 近取诸身, 远取诸物, 于是始作八卦, 以通神明 之德, 以类万物之情”(《易. 系辞下》)。其“造书契以代结 绳之政, ......以俪皮为礼, 结网罟以教佃渔”(唐. 司马贞《补 史记三皇本纪》), 教世人识礼仪, 使社会井然有序。与女 娲相比, 这位伟大的男神身上人性显现的更多一些。
我们看到的伏羲像一位智者, 他从感悟天地自然规律 中寻找到了社会文明发展的钥匙, 并对他的子民倾囊相授, 这种情操和胸襟正是后代诸多先贤圣哲终其一生所追求和 向往的。

伏羲造书契、作八卦、教佃渔等事, 其实都是发生在 父系社会中人类的发明创造。考古学家在半坡、柳湾等地 出土的陶器上发现了一种介于图画与文字之间的记事符 号, 在山东莒县陵阳河和诸城前寨两个大汶口文化遗址中, 发现了一些图象文字, 唐兰先生通过研究说: “这些象形文 字跟商、周青铜器文字、商代甲骨文字以及陶器文字, 都 是一脉相承的。”[7]这恐怕是伏羲造书契的历史真实吧。

八卦, 是华夏先民特殊的思维模式, 其中所反映的对立统 一思想, 构成了华夏民族独特的认知模式和审美心态。善 良的远祖先民将所有的荣耀与功绩都归属于这位男性神, 其目的无疑是为了提升他在神谱中的地位与份量。于是伏 羲王天下, 女娲辅治国。

\section{4. 女娲、伏羲关系演变的文化学阐释}

母系社会向父系社会发展, 使男性逐渐在社会中取得 主导地位, 并最终取代女性成为社会的主宰。地位的改变, 使男性要求在精神领域里提高自身地位, 所以神话世界中 必然会有所变化: 众神在不知不觉中由女性渐渐过渡为男 性。然而母亲却无法替代, 她的功绩和伟大不容否认, 所 以唯有作为母亲神的女娲, 因此逃过了被“变性”的劫难。 但其地位却每况愈下, 由创世的唯一女神, 降为伏羲的助 手、妹妹、妻子。这一变化也显示出女性在社会生活中已 从领导统治地位下降至从属地位, 可见, 男性大神的出现 是历史发展的必然。

纵观有关这女娲、伏羲两位神祇的文字资料, 不难发 现, 在秦汉之前的资料中, 女娲和伏羲是各自独立的两位 大神, 他们之间相互并没有什么关系, 且各自有着自己的 神话功绩。但是, 在汉代, 就出现了许多有关二者关系的 记录, 汉代的许多石刻画、砖壁画都呈现两者交尾的状态, 隐约吐露着二者夫妻的关系。与此同时, 女娲的地位也有 所改变: 《淮南子. 览冥训》记: “女娲, 阴帝, 佐虙戏治者 也”, 将女娲归为伏羲的治国助手; 《路史. 后纪二》注引汉 代 《风俗通》说: “女娲, 伏希 (羲) 之妹”; 唐人卢全又 说: “女娲本是伏羲妇”(唐. 卢全 《与马异结交》); 直到唐 代李呪手里, 女娲和伏羲的关系才被完整地描绘为兄妹夫 妻: “昔宇宙初开之时, 有女娲兄妹二人, 在昆仑山, 而天 下未有人民。议以为夫妻, 又自差耻。兄即与其妹上昆仑 山, 咒曰: ‘天若遣我二人为夫妻, 而烟悉合; 若不, 使烟 散。'于烟即合。其妹即来就兄。”(唐. 李圥《独异记》)

其实, 女娲、伏羲婚配的神话, 可以归为推原神话。 
所谓“推原, 就是推寻事物的开始、起源。这是由于初民对 事物的成因每每不能解释, 只好创造神话以解释之, 以满 足其求知欲望和属于人类本能的好奇心。”[8]就如同神话 中有关于女如补天之事, 女娲炼五色石补天, 但是天为什 么会被破坏, 神话中没有说明。于是人们又创造了一则神 话来解释, 共工怒撞不周山, 使“天柱折, 地维缺”, 所以 女娲要补天救民。同样, 当先民对生育有了更明确的概念 和认识之后, 女娲抟黄土造人就不能再满足人类起源之说, 于是他们又创造出女娲、伏羲结合成为人类共同的创造者。 因为神话中所述之事, 在先民看来是真实存在过的, 当女 娲造人神话存在的真实性发生动摇时, 人们有必要重新创 造一个神话去补充和完善整个神话系统, 从而保持其在先 民中的可信性。

至于唐人李冗所记载的女娲、伏羲的故事, 显然已是 几经加工的 (它借鉴和吸收了瑶族等少数民族的神话传 说), 其中可能保存着一些古神话的影子, 但更多的融入了 后人的理性思考和后世的社会人文色彩: 故事中二人 “议以 为夫妻, 又自差耻”, 此“差耻”二字显然是封建礼教熏陶的 结果。在封建社会中, 没有媒妁之言、父母之命而自议结 合的婚姻是被社会所不齿的, 也是礼教所不能容忍的, 可 见作者是将其受之文化过渡给了女娲伏羲二人。差耻之心 可以说是人类迈向文明的重要标志: 在蛇诱惑之前, 夏娃 与亚当赤裸裸地生活在伊甸园中, 并没有感到过差耻。智 慧与欲望往往是并生的, 欲望使他们抵不住蛇的诱惑吃了 智慧果, 智慧使他们为赤裸的自己感到差愧难当, 同时也 使人类开启了通向文明的大门。

兄妹成婚在古代婚姻史上确实存在过, 而且持续时间 相当长久, 其影响力也是极久远的。在中外许多文学作品 中, 我们都可以很容易找到例证; 而今天, 在某些偏远的 山区依然存在兄妹成婚的情况。在人类婚姻史上, 原始社 会早期, 人类曾历过混婚制、伙婚制, 这都是兄妹婚配的 婚姻制度 [9], 且男女的结合往往比较自由随意。女娲伏羲 兄妹成婚的神话应是对上古这两种婚姻制度的一种朦胧的 追忆。

在有关女娲、伏羲的资料中, 有一点很有趣: 所有的 文字和图像都显示他们具有相同的形体, 都是人首蛇身, 如果模糊其性别的差异, 他们则完全是同一种东西。闻一 多先生曾用音韵训诂学的方法考证了伏羲、女娲的读音, 他认为伏羲又写作 “包戲” (较古的写法), 在古代读音和 “匏 瓠”相同, 意为葫芦; “女娲”又叫做“(1)娲”, 在古代读音和 “湤瓜”相同, 亦指葫芦。所以闻先生说: “伏羲与女娲, 名 虽二, 义实只一。二本皆为葫芦的化身, 所不同者, 仅性 别而已。称其阴性的曰 ‘女娲', 犹言 ‘女匏 (2) 、“女伏羲” 也。”[10]闻老意说二者皆为葫芦 (或葫芦㼼), 女娲就是
伏羲。其实我们不妨把此说反过来推, 伏羲就是女娲, 即 男女娲, 二者实为一物。伏羲是神谱世系转变时, 女娲神 由女性向男性转变的产物。女如相比较其他诸女神是幸运 的, 没有从神谱中消失, 且依然稳固地保持着她的功绩和 神性, 并与伏羲一同流传于后世。

\section{5. 结束语}

社会的变迁给神话世界带来了“翻天覆地”的变化, 改 变了中国神话系统中神性结构。这种变革的出现恰恰说明 了中国神话的原始性, 原始的神话应该是质朴的、松散的, 所以易受到外界的影响。中国神话虽然没有希腊神话那样 完整的结构和系统, 但其却尽可能的保持着神话最原始的 风貌, 以待后人去探求其中的奥妙。

注释:

(1)bāo 左右结构 女加包

(2)xì 左右结构 戲将戈旁换为瓜旁

\section{参考文献(References)}

[1] Karl Marx, "Marx and Engels, Selected Works - Introduction of Critique of Political Economy," (Second Edition) Central Compilation and Translation Press, vol. 2, 1995, pp. 29.

[2] Wu Tianming, "Chinese mythology research," Central Compilation and Translation Press, 2003, pp. 7.

[3] Wang Yuzhe, “Ancient Chinese History," Shanghai People's Publishing House, 2000, pp. 54.

[4] Wang Yuzhe, "Ancient Chinese History," Shanghai People's Publishing House, 2000, pp. 74.

[5] Liao Qun, "Chinese aesthetic cultural history-Pre-Qin volume," Shandong Pictorial Publishing House, 2000, pp. 50.

[6] Morgan, Yang Chun translation, "Ancient Society," Jiangsu Education Publishing House, 2005, pp. 318.

[7] Tang Lan, "Preliminary Exploration of cultural sites and Written Characters about Jiangxi Wu city," Cultural Relics, 1975, pp. $72-76$.

[8] Yuan Ke, “Ancient myths interpretation," People's Literature Publishing House, 1979, pp. 32.

[9] Morgan, Yang Chun translation, "Ancient Society," Jiangsu Education Publishing House, 2005, pp. 339.

[10] Wen Yiduo, "Mythological Studies- Fu Xi research," Bashu Publishing House, 2002, pp. 109-110. 